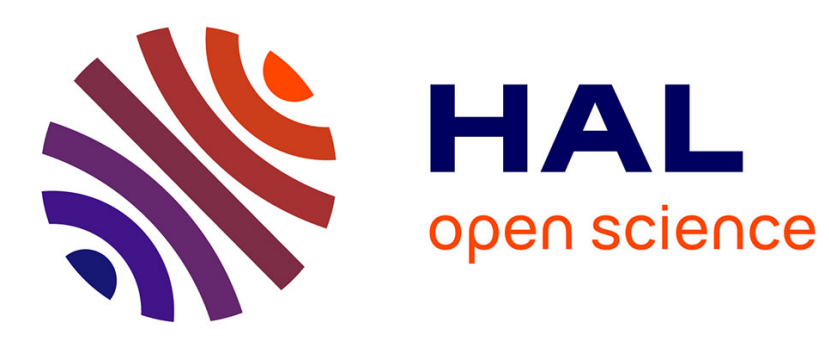

\title{
Evolutionary Mesh Numbering: Preliminary Results
}

Francis Sourd, Marc Schoenauer

\section{To cite this version:}

Francis Sourd, Marc Schoenauer. Evolutionary Mesh Numbering: Preliminary Results. Adaptive Computing in Design and Manufacture, ACDM'98, Apr 1998, Plymouth, pp.137-150. inria$00153379 \mathrm{v} 2$

\section{HAL Id: inria-00153379 https://hal.inria.fr/inria-00153379v2}

Submitted on 11 Jun 2007

HAL is a multi-disciplinary open access archive for the deposit and dissemination of scientific research documents, whether they are published or not. The documents may come from teaching and research institutions in France or abroad, or from public or private research centers.
L'archive ouverte pluridisciplinaire HAL, est destinée au dépôt et à la diffusion de documents scientifiques de niveau recherche, publiés ou non, émanant des établissements d'enseignement et de recherche français ou étrangers, des laboratoires publics ou privés. 


\title{
Evolutionary Mesh Numbering: Preliminary Results
}

\author{
Francis Sourd and Marc Schoenauer \\ CMAP - URA CNRS 756 \\ Ecole Polytechnique \\ Palaiseau 91128, France \\ marc.schoenauer@polytechnique.fr
}

in I. Parmee, ed., Proceedings of ACDM'98, pp 137-150, Springer Verlag, 1998

\begin{abstract}
Mesh numbering is a critical issue in Finite Element Methods, as the computational cost of one analysis is highly dependent on the order of the nodes of the mesh. This paper presents some preliminary investigations on the problem of mesh numbering using Evolutionary Algorithms. Three conclusions can be drawn from these experiments. First, the results of the up-to-date method used in all FEM softwares (Gibb's method) can be consistently improved; second, none of the crossover operators tried so far (either general or problem specific) proved useful; third, though the general tendency in Evolutionary Computation seems to be the hybridization with other methods (deterministic or heuristic), none of the presented attempt did encounter any success yet. The good news, however, is that this algorithm allows an improvement over the standard heuristic method between $12 \%$ and $20 \%$ for both the 1545 and 5453 -nodes meshes used as test-bed. Finally, some strange interaction between the selection scheme and the use of problem specific mutation operator was observed, which appeals for further investigation.
\end{abstract}

\section{Introduction}

Most Design Problems in engineering make an intensive use of numerical simulations of some physical process in order to predict the actual behavior of the target part. When the mathematical model supporting the numerical simulation involves Partial Differential Equations, Finite Element Methods (FEM) are today one of the most widely used method by engineers to actually obtain an approximate numerical solution to their theoretical model. However, the computational cost of up-to-date numerical methods used in FEM directly depends on the way the nodes of the underlying mesh (i.e. the discretization) are numbered. Solving the Mesh Numbering Problem (MNP) amounts to find the permutation of the order of the nodes that minimizes that computational cost.

Numerical engineers have developed a powerful heuristic technique (the socalled Gibb's method) that gives reasonably good results, thus providing a clear reference to any a new mesh numbering algorithm. 
The goal of this paper is to use Evolutionary Algorithms (EAs) to tackle the MNP. EAs have been widely applied to other combinatorial optimization problems, among which the popular TSP 10.4. However, as far as we know, this is the first attempt to solve the MNP using EAs. Unfortunately, though both problem look for a solution in the space of permutations of $[0, n]$, the specificity of the MNP might make inefficient the simple transposition of TSP techniques to the MNP. Indeed, looking at the history of Evolutionary TSP, it seems clear that the key of success is hybridization with problem-specific techniques: from the Grefenstette's early incorporation of domain knowledge 10 to the most recent works [4, 12,6] where evolutionary results can - at last - be compared to the best Operational Research results, even for large size TSP instances. So the path to follow here seems rather clear: design some NMP-specific operators, and compare their performances to either "blind" problem independent operators or TSP-specific operators.

The paper is organized as follows: Section 2 recalls the basics of Finite Element Methods, and precisely defines the objective function. The state-of-the-art "Gibbs method' is also briefly described. Section 3 presents the design of the particular Evolutionary Algorithm used thereafter, discussing in turn the representation issue, specific crossover and mutation operators, and the initialization procedure. This algorithm is then experimented in section 4, with emphasis on the tuning of the probabilities of application of all operators at hand (both problem-specific and problem independent). First, the crossover operators all rapidly appear harmful. Second, surprising interactions between the selection scheme and the different mutation operators seem to indicate that, though at the moment domain knowledge did not increase the performances of the algorithm, there is still some room for improvement in that direction. Finally, the usefulness of evolutionary mesh numbering with respect to Gibbs' method is discussed: the results are indeed better, but the cost is also several orders of magnitude greater.

\section{Mesh Numbering}

\subsection{Theoretical Background}

Many models for physical, mechanical, chemical and biological phenomenon end up in Partial Differential Equations (PDE) where the unknown are functions defined on some domain $\Omega$ of $\mathbb{R}^{n}$.

A popular way to transform a system of PDEs into a finite linear system is the Finite Element Method (FEM) 18, 3]. The domain $\Omega$ is discretized into small elements, who build up a mesh. A typical mesh - on a non-typical domain - is given in Figure 1. The solution of the initial PDEs is sought in spaces of functions that are polynomial on each element. Such an approximate solution is completely determined by its values at some points of each element, called the nodes of the mesh. Those values are computed by writing the original PDEs locally on each element, resulting in a linear system of size the number of nodes times the number of degrees of freedom, or unknown values, at each node). Usual sizes 
for such systems range from a few hundreds (e.g. in two-dimensional structural mechanics) to millions (e.g. for three-dimensional problems in aerodynamics).

However, as a consequence of the local discretization, the equation at each node only involves values at a few neighboring nodes: the resulting matrix is hence very sparse. And specific methods exist for sparse system [9], whose complexity is proportional to the square of the bandwidth of the matrix, i.e. the average size of the profile of the matrix, given by the sum over all lines of the maximal distance to the diagonal of non-zero terms. For full matrices, the bandwidth is $n(n-1) / 2$ while it is $n$ for tridiagonal matrices (for $n \times n$ matrices).

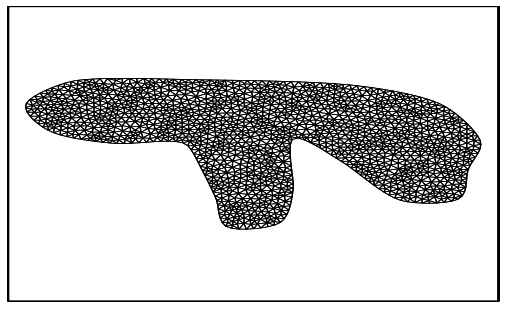

Fig. 1. Sample mesh with 1545 nodes.

\subsection{Computing the bandwidth}

The contribution of each single line to the total bandwidth of the matrix is highly dependent on the order of the nodes of the mesh: the equation for node number $i$ only involves the neighboring nodes; hence the only non-zero terms of the corresponding equation will appear in the matrix in the column equal to the number of the node in the mesh. Depending on the order of the nodes in the mesh, the bandwidth can range from a few units to almost the size of the matrix.

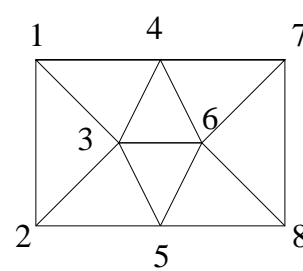

(a) Bandwidth=18

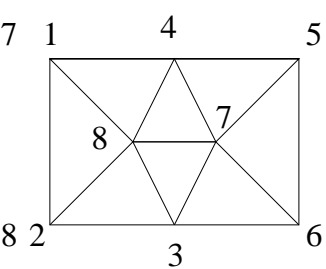

(b) bandwidth=26

Fig. 2. A simple example of bandwidth with respect to nodes order.

A simple example of a mesh is given in Figure 2 for a two-dimensional domain discretized into triangles. The nodes of that mesh are the summits of the 
triangles (note that, while common, this situation is not the rule, and the middle of the edges, the center of gravity of the triangles, ... can also be nodes). The effect of numbering is demonstrated in Figures 2 a and 2 -b, where the same mesh can give a bandwidth of 18 or 26 , depending on the order in which the nodes are considered.

The only useful data for mesh numbering is, for each node, the list of all neighbors. For instance, the mesh of Figure 2 a can hence be viewed as

$\left(\begin{array}{lll}2 & 3 & 4\end{array}\right)\left(\begin{array}{lll}1 & 3 & 5\end{array}\right)\left(\begin{array}{lllll}1 & 2 & 4 & 5 & 6\end{array}\right)\left(\begin{array}{llll}1 & 3 & 6 & 7\end{array}\right)\left(\begin{array}{llll}2 & 3 & 6 & 8\end{array}\right)\left(\begin{array}{lllll}3 & 4 & 5 & 7 & 8\end{array}\right)\left(\begin{array}{lll}4 & 6 & 8\end{array}\right)\left(\begin{array}{lll}5 & 6 & 7\end{array}\right)$

Once an order for the nodes is chosen, the profile of the matrix can be constructed: an upper bound ${ }^{1}$ of the fitness function is given by the following equation (11), where $N$ is the number of nodes, and, for each $i$ in $[1, N], \mathcal{N}(i)$ is the set of neighbors of node $i$ :

$$
\mathcal{B}=\sum_{i=1}^{i=N} \min _{(j>i) ;(j \in \mathcal{N}(i))}(j-i)
$$

Note that due to symmetry, only the contribution to the bandwidth of the upper part of the matrix is considered.

For the simple case of the mesh of figure 2, the bandwidth is $3+3+3+3+$ $3+2+1=18$ for the order (a) and $7+6+5+4+2+1+1=26$ for the order (b).

The goal of mesh renumbering is to find the order of the nodes (i.e. a permutation on $[1, N])$ that minimizes the bandwidth $\mathcal{B}$.

\subsection{State of the art}

The problem of mesh numbering is clearly a $N P$ - complete combinatorial problem, as the search space is the space of permutations in $[1, n]$. Hence, no exact deterministic method can be hoped for. Numerical scientists have paid much attention to that problem, developing heuristic methods. The favorite method nowadays, used in most FEM software packages, is the so-called Gibbs method presented in detail in [5]). It performs three successive steps, and use the graph $G$ representing the "neighbor to" relationship between nodes.

- Find both ends of the numbering. They are chosen such that, first, their distance (in term of minimal path in $G$ joining them) is maximal, and second, each one has the lowest possible number of neighbors.

- Make a partition of graph $G$ into layers containing nodes at the same distance from the origins.

- Number the nodes breadth-first, starting from one origin (i.e. numbering nodes layer by layer).

\footnotetext{
${ }^{1}$ Some of these candidates to be non-zero can actually be null, depending on the actual formulation of the PDEs. But this rare eventuality will not be considered in the general framework developed here.
} 
As Gibbs method is used in all FEM packages, the minimum requirement for any other algorithm to be considered interesting by numerical scientists is to obtain better results than those of Gibbs. Hence, in the following, all results of performance will be given relatively to those of Gibbs.

\section{The evolutionary algorithm}

\subsection{MNP is not a TSP}

Combinatorial optimization is a domain where evolutionary algorithms have encountered some successes, when compared to state-of-the-art heuristic methods. Probably the most studied combinatorial optimization problem is the Traveling Salesman Problem (TSP). In both cases (MNP and TSP), the search space is that of all permutations of $N$ elements.

However, a first obvious difference is that both the starting point and the "direction" of numbering are discriminant in the MNP while they are not in the TSP. As a consequence, the size of the search space in the MNP is $n$ ! while it is $(n-1) ! / 2$ for the TSP. On the other hand, no degeneracy (see [14) is present in the permutation representation for the MNP (see below).

Second, the MNP is not so easily decomposable. A whole part of a solution of the TSP can be modified without modifying the remaining of the tour. In the MNP on the other hand, the propagation of any modification has consequences on all geometrical neighbors of all modified nodes.

As a consequence, the useful notion of "neighbor" is totally different from one problem to another: In the TSP, two towns are usually called neighbors if they are visited one after the other on the tour at hand; In the MNP, the "neighbor of" relationship is absolute geometrical data, independent of any order.

Practically, a major difference between both problems is the absence of any local well known optimization algorithm for the MNP: as quoted in the introduction, the best results obtained so far by evolutionary algorithms on the TSP are due to hybrid "memetic" algorithms searching the space of local optima with respect to a local optimization procedures (e.g. the 2-opt or 4-opt procedures in [4, 12,6]). Such strategy cannot be reproduced for the MNP.

\subsection{Representation}

One important consequence of these differences is that the concepts of "edges" or "corners" [14], known to be of utter importance for the TSP, do not play any role in the MNP.

Two representations will be experimented with in that paper:

- In the permutation representation, a permutation is represented by the sequential list of the numbers of all nodes. Note that this representation relies on a predefined order of the nodes (corresponding to the identity permutation $(1,2,3, \ldots, N))$. Some consequences are discussed in section 3.5. 
- All permutations can be decomposed in a sequence of transpositions. Moreover, all sequences of transpositions make a unique valid permutation. The transposition representation describes a permutation as an ordered list of transpositions. Note that this representation is highly degenerate: many genotypes correspond to the same phenotype.

\subsection{Crossover operators}

Four crossover operators have been tested, from general-purpose operators to MNP-specific crossovers.

- The transposition crossover is a straightforward crossover for the transposition representation: It exchanges portions of the transposition lists of both parents. As any combination of transpositions make a valid permutation, it directly generates valid permutations.

- The edge crossover is a general-purpose operator for permutation problems, designed and tested on the TSP problem [17]. No specific knowledge about the problem is used, but the underlying assumption is that edges are the important features in the permutations. All experiments using the edge crossover gave lousy results, thus confirming that edges do not play for the MNP the important role they have in the TSP. That crossover will not be mentioned any more here.

- The breadth-first crossover is based on the heuristic technique described in section 2.3, but uses additional information from both parents to generate one offspring.

A starting point is randomly chosen, is given the number it has in parent $A$, and becomes the current node. All neighbors of the current node are numbered in turn, being awarded an unoccupied number, before being put in a FIFO stack. To number the neighbor $N$ of a node $M$ already numbered $i_{M}$, the differences $\Delta_{A}$ and $\Delta_{B}$ of the numbers of nodes $M$ and $N$ respectively in parent $A$ and parent $B$ are computed. If the number $i_{M}+\min \left(\Delta_{A}, \Delta_{B}\right)$ is free, it is given to node $N$. Otherwise, if the number $i_{M}+\operatorname{Max}\left(\Delta_{A}, \Delta_{B}\right)$ is free, it is given to node $N$. Otherwise, the number closest to $i_{M}$ which is not yet used is given to node $N$.

All nodes are processed once, and are given a yet-unattributed number, generating a valid permutation. This crossover tries as much as possible to reproduce around each node the best local numbering among those of both parents.

- The difference crossover was designed to both preserve the diversity in the population and try to locally minimize the fitness function. From both permutation representations of the parents, all nodes having the same number are given that common number in the offspring permutation. Further, all remaining nodes in turn (in a random order) are given the number which is the closest possible from all its neighbors numbers. 


\subsection{Mutation operators}

Here again, both general-purpose and problem specific mutation operators were tested.

- The minimal mutation for the permutation representation is the exchange of numbers between two randomly chosen nodes (i.e. the application of a transposition), thereafter termed transposition mutation. Its strength can be adjusted by repeated application during the same mutation operation.

- The neighbor transposition mutation is a slight modification of the above operator: after the first node has been chosen randomly, it is exchanged with one of its neighbors.

- The neighbor permutation mutation is a further step in the direction of using neighbor information to perform mutation: a node is randomly chosen, and a random permutation among the numbers of all its neighbors is performed.

- The inversion mutation reverts some part of the permutation. A special case of the inversion mutation is when the whole permutation is inverted. This operator was found useful in Gibbs method (section 2.3).

- The choice of the origin of the numbering is important in the MNP. The origin mutation was designed to handle this issue. An integer $i$ is randomly chosen, and all numbers $j$ are replaced by either $i+j \bmod n$ either $n-$ $(i+j) \bmod n$, on a local minimization argument (the brute translation only gives birth to usually very bad numbering when the old origins met).

\subsection{Initialization procedure}

The standard way to initialize a population is to perform a uniform sampling of the genotype space. However, alternative specific ways have been proposed: for instance, the greedy heuristic for the TSP (from a random initial town, chose the nearest town not yet visited) constructs individuals with a tour length of about $20 \%$ more than the optimal value on average 㕵. In the same line, three different initialization procedures have been tested for the MNP.

- The standard uniform sampling of the standard representation (section 3.2) was the first obvious choice. It is termed random initialization.

- As the state-of-the-art solution is given by the output of the Gibbs method (section 2.3), the Gibbs initialization performs only slight perturbations of the Gibbs solution to generate the initial permutations. This is achieved using the transposition representation (section 3.2) taking the Gibbs order as reference, and allowing only a small number of transpositions. Note that in this case, the original Gibbs result is included as the first individual of the population.

- The trouble with the above Gibbs initialization is a very strong bias toward solution quite similar to Gibbs. If higher optima are located in very different regions of the permutation space, they will probably not be found. To address that issue, the point initialization was designed, based on some breadth-first heuristic similar to Gibbs', but using a random starting point (Gibbs process is very sensitive to the choice of the initial point). 
The average bandwidth of permutation drawn using the uniform initialization is of course quite large (more than 6 times that of Gibbs method). The point initialization gives much better individuals: their bandwidth range from $20 \%$ to $100 \%$ above Gibbs results while Gibbs initialization stays between $0 \%$ and $15 \%$ above Gibbs order.

\section{Experimental Results}

\subsection{The meshes}

Three meshes have been used to test the algorithm described above: the small mesh has 164 nodes, the medium one has 1544 nodes (see Figure 11) and the large mesh has 5453 nodes. The computational cost of one fitness function evaluation increases linearly with the size of the mesh. Hence, most initial experiments were performed in the small mesh. The validation of clear tendencies was then carried on for confirmation on the medium mesh. The best combination of parameters were finally tested on the large mesh, as its size is becoming to be of some interest for real world application.

\subsection{Experimental settings}

Two basic evolution schemes were used: a standard GA, with linear ranking and elitist generational replacement; a $(\mu+\lambda)$-ES scheme, in which all $\mu$ parents give birth to $\lambda$ offspring, the best $\mu$ of the $\mu+\lambda$ parents + offspring becoming the parents of the next generation. The first series of experiments were performed on the mesh, using a population sizes of 50 for the GA scheme, and a $(7+50)$-ES. Both schemes were tested with the same combinations of $P_{c}-P_{m}$, (crossover rate - mutation rate). If an individual undergoes crossover, a mate is selected and only one offspring is generated. One single type of crossover was made possible at each run. The resulting offspring (or the initial individual if no crossover occurred) then undergoes mutation with probability $P_{m}$. If mutation happens, one mutation operator is chosen according to user-defined weights, and, in the case of transposition mutations, one single transposition is performed.

\subsection{First tuning on the small mesh}

As said above, intensive experiments were performed on the small mesh. During those experiments, all values for $P_{c}$ and $P_{m}$ between 0 and 1 by 0.1 steps were tried independently, for all possible crossover operators. Those runs were allowed 150000 fitness evaluations (unless otherwise mentioned).

Preliminary runs were performed to tune the mutation weights ( 16 $)$. The weights for the 5 mutations were first set equal. Then a close look at the types of mutations that the best individual in the population was submitted to, along different runs, allowed to eliminate both the inversion mutation and the origin 
mutation. Only the random transposition mutation, the neighbor transposition mutation and the neighbor permutation mutation proved useful, and their weights PmRand, PmNeighbor and PmAround were set to values between 0 and 1, their sum being equal to 1 .

Initialization procedures As could be predicted, the best on-line results were obtained for the Gibb's initialization, as its starting point was rather better than both other. But whereas the point initialization almost caught up (as will be seen in forthcoming section 4.4), the random initialization stayed far beyond, even when allowed ten times the number of fitness evaluations. So only the Gibb's and the point initializations will be considered in the following.

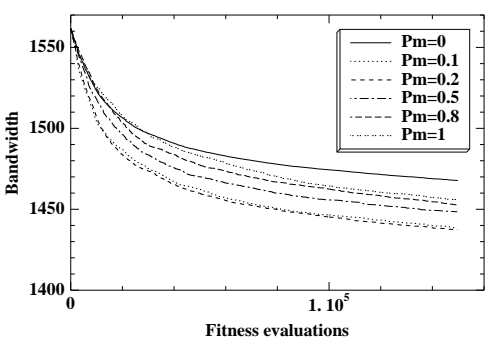

(a) GA with breadth-first crossover

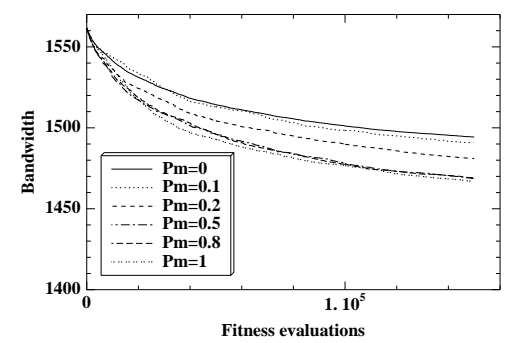

(c) GA with transposition crossover

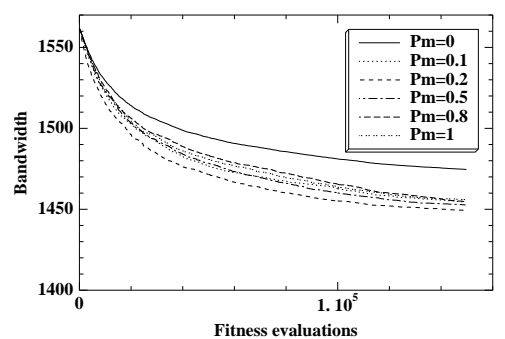

(b) GA with difference crossover

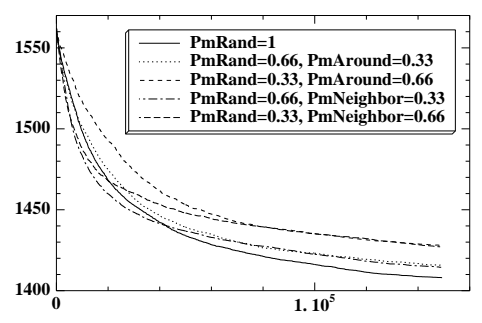

(d) $(7+50)-E S-$ no crossover

Fig. 3. Typical on-line results (averages over 21 runs) on the small mesh: for the three $G A$ runs, population size is 50, $P_{c}$ is 0.6 and $P_{m}$ is as indicated; for the (7+50)-ES runs, $P_{m}=1$; in all cases, whenever an individual is mutated, PmRand $=0.66$ and PmNeighbor $=0.33$.

Crossover operators and evolution schemes The first experiments aimed at comparing the crossover operators, and adjusting the crossover rate $P_{c}$. A common feature could be observed for all three crossovers: when using the GA scheme, and except for high values of $P_{m}$, for which it made no significant difference, the results decreased when $P_{c}$ decreased from 1 to 0.6 or 0.5 . Moreover, 
and almost independently of the settings of the mutation weights, the best results were obtained with the $(7+50)$-ES scheme, with $P_{c}=0$ (higher values of $P_{c}$ for the ES scheme performed rather poorly - but this might be because of the rather small population size of 7 ).

When it comes to compare the crossover operators, the results of Figure 3 (a), (b) and (c), are what could be expected: the "blind" transposition crossover (c) performs rather poorly - and gets its best results for the highest values of $P_{m}$ which seems to indicate that it is really not helping much. On the opposite, both other operators, that do incorporate some domain knowledge, get their highest performances for $P_{m}=0.2$ and $P_{m}=0.1$. Moreover, the breadth-first crossover (a) performs better than the difference crossover (b) (and the difference is statistically significant with $99 \%$ confidence T-test after 300000 fitness computations).

A last argument favoring the abandon of crossover operators is the extra cost they require, as based on local optimization heuristics of complexity $o(N)$. For instance the total CPU time is increased by a factor around 4 between runs with $P_{c}=0$ and $P_{c}=1$ (from 3 to $13 \mathrm{mn}$ on average for 300000 evaluation runs on a Pentium P200).

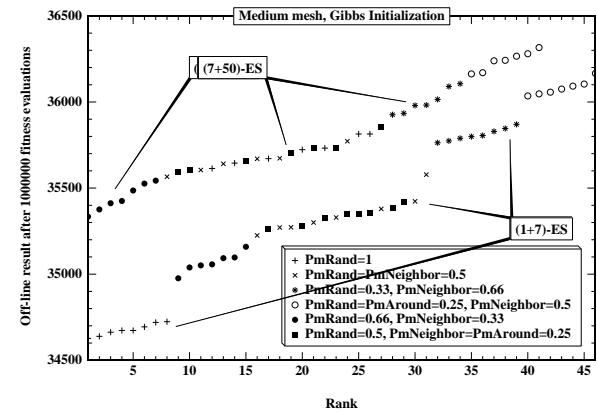

(a) Gibbs initialization

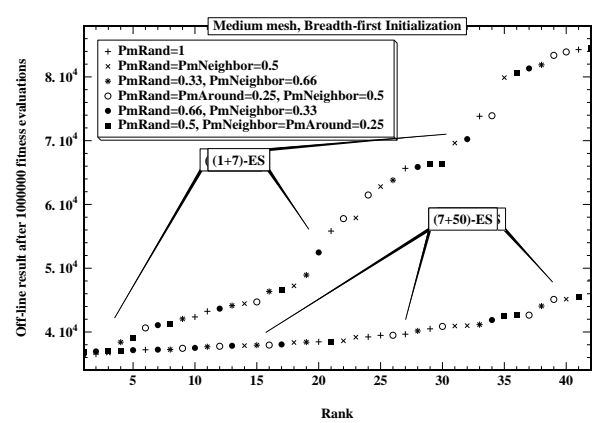

(b) Breadth-first initialization

Fig. 4. Off-line results of both (7+50)-ES and (1+7)-ES after 1000000 fitness evaluations, for different settings of the mutation weights.

\subsection{Mutations and population size}

This section presents some results obtained on the medium and large meshes, using mutation operators only inside some $E S$ evolution scheme. At first, the goal of these experiments was to sort out the usefulness of problem-specific knowledge, in the initialization procedure and in the mutations operators. Bur it rapidly turned out that the population size also was a very important parameter.

Figure 4 witnesses the surprising results obtained on the medium mesh: each dot indicates the best fitness reached after 1 million fitness evaluations of a single run of the evolutionary algorithm. The different shapes of the dots represent different settings of the relative mutation rates PmRand, PmNeighbor and 
PmAround. Figure 4 a shows the runs that used the Gibbs initialization procedure while Figure $4 \mathrm{~b}$ those who used the breadth-first initialization procedure. On each Figure, the two distinct sets of points correspond to the $(1+7)$ - and the $(7+50)$-ES schemes, as indicated. Note that all trials with larger population sizes were unsuccessful.

Some clear conclusions can be drawn from these results. First, the overall best results are obtained for the $(1+7)$-ES scheme starting from the Gibbs initialization and using the "blind" transposition mutation only (Figure 4-a) (see section 4.5 for a detailed comparison with the results of Gibbs method). But comparing the results between Gibbs and breadth-first initialization procedures on the one hand, and $(1+7)$ - and $(7+50)$-ES schemes on the other hand gave some unexpected results:

- Whereas the $(1+7)$-ES scheme consistently outperformed the the $(7+50)$-ES scheme when using Gibbs initialization, the reverse is true for the breadthfirst initialization;

- Whereas different settings of the mutation rates gave rather different results for the Gibbs runs, this does not seem to be so clear for the breadth-first runs;

- whatever the initialization, the $(7+50)$ results are more stable than the $(1+7)$ results with respect to mutation rates; This is striking on the breadth-first plot, but also true on the other plot.

- the worst results are obtained for the lowest values of PmRand (the results with even lower values of PmRand are not presented here, but were very poor). However, domain specific mutation operators (see the black circles and squares, compared to the "+" dots) are more efficient with the $(7+50)$ scheme than with the $(1+7)$ scheme. This is specially visible on the Gibbs runs.

Note that these tendancies were confirmed when the runs were allowed more fitness evaluations (e.g. 10 millions, see forthcoming section 4.5).

Some tentative explanations can however be proposed, after noticing that the $(1+7)$ scheme can be viewed more like a depth-first algorithm while the $(7+50)$ scheme searches in a more breadth-first manner.

So, using Gibbs initialization probably tights the population in a very limited area of the search space from which it is useless to try to escape: this favors the performance of depth-first search, as breadth oriented search does not have the possibility to jump to other promising regions. Moreover, it seems that the transpositions of neighbor nodes in a depth-first search does not allow large enough moves to easily escape local optima, resulting in the best results for the pure random mutation for the $(1+7)$.

On the other hand, successive local moves have greater chances of survival in the $(7+50)$ breadth search, and give the best results in that case (though some random mutation are still needed). And when it comes to a more widely spread population after the breadth-first initialization, the breadth-first search demonstrates better results by being able to use more efficiently in that case the 
domain neighboring information.

This situation suggests further directions of research: First, the $(1+7)$-ES scheme with pure random mutation resembles some sort of Tabu search [7, 8], and so might be greatly improved by adding some memory to it, either deterministically, like in standard Tabu search, or stochastically, as proposed in [15, 13 .

Second, more than one neighbor transposition seems necessary to generate improvements. Hence, the number of transpositions should not be forced to 1 , and can be made either self-adaptive, with the same problems than in the case of integer variables in Evolution Strategies [1], or exogenously adaptive, as proposed in [2] where some hyperbolic decreasing law is used for the mutation rate along generations.

\subsection{Evolutionary mesh numbering vs Gibbs method}

But apart from optimizing the evolutionary algorithm itself on the MNP, a critical point is whether evolutionary mesh numbering can compete with the Gibbs method. Of course, if the Gibbs initialization is used, as the original Gibbs numbering is included in the initial population, any improvement is in fact giving a better result than the Gibbs method. But how interesting is that improvement, especially when the computational cost is taken into account?

On the medium mesh (1545 nodes), the bandwidth using Gibbs method is 39055. As can be seen on Figure 1 , the best result of the $(1+7)$-ES with pure random mutation after 1 millions evaluations is 34604, i.e. an improvement of $11.4 \%$. The computational cost of one run is $15-20 \mathrm{mn}$ (on a Pentium $200 \mathrm{Mhz}$ Linux workstation), to be compared to the 20 s seconds of Gibbs method!

If the maximum number of function evaluations is set to 10 millions, the best result in the same conditions is 32905 (i.e. $15.75 \%$ improvement), with an average over 21 runs of $33152(15.11 \%)$. Of course, in that latter case, the computational cost is 10 times larger ...

The results on the large mesh (5453 nodes) follow the same lines, though only the combinations of parameters found optimal on the medium mesh were experimented with, due to the computational cost (around 12-14 hours for 10 Millions evaluations).

From a Gibbs bandwidth of 287925 (obtained in about one minute), the best result for the $(1+7)$-ES with only random mutation was $257623(10.52 \%)$ while the average of 6 runs was $258113(10.35 \%)$. On the other hand, the best for $(7+50)$-ES was $262800(8.73 \%)$, the average being $263963.43(8,32 \%)$, with PmRand $=0.66$ and PmVois $=0.33$ (best parameters of Figure 4 a).

The first a priori conclusion is that the computational cost of the evolutionary algorithm makes it useless in practical situations: an improvement of 
between 10 and $15 \%$ requires a computational power of several order of magnitude larger. This quick conclusion must however be moderated: First, due to the quadratic dependency of the computational cost of the matrix inversion in term of the bandwidth, the actual gain in computing time is around $35 \%$ for a $15 \%$ bandwidth decrease. And second, many meshes used nowadays in industry require a few months of manpower to be built and validated. So 24 more hours of computation for a better numbering is relatively low increase in cost. And if the mesh is then put in an exploitation environment, and is used in several thousands of different Finite Element Analyses, then the overall gain might be in favor of getting a really better numbering, even at what a priori seems a high computational cost. But of course this means that meshes of up to a few thousands nodes can be handled by evolutionary algorithms.

It is nevertheless important to notice that the computation of the bandwidth can be greatly optimized. The present algorithm was designed to be very general, handling any possible operator. Hence it always computes the fitness from scratch. However, in the case where only a small number of transpositions are performed, the variation of the fitness could be computed, by examining only the neighbors of the transposed nodes.

\section{Conclusion}

We have presented feasibility results for the application of Evolutionary Computation to the problem of mesh numbering. Our best results outperform the state-of-the-art Gibbs method by 10 to $15 \%$ on the two test meshes used (with 1545 and 5453 nodes respectively). Whereas these sizes would appear fairly high for TSP problems for instance, they are still small figures with respect to realworld problems, where hundreds of thousands of nodes are frequent.

From the Evolutionary point of view, two issues should be highlighted. First, though both general-purpose and domain-specific crossover operators were tried, none proved efficient. A possible further trial could be to use more global geometrical information (e.g. divide the mesh into some connected components, and exchange the relative orders of such blocks, in the line of [11]).

Second, the overall best results were obtained by a $(1+7)$-ES using pure random transposition mutation and starting from an initial population made of slightly perturbed Gibbs meshes. This which might be an indication that other heuristic local search methods (e.g. Tabu search) might be better suited to the MNP. However, as discussed in section 1 , some hints make us believe that there is still a large room for improvement using evolutionary ideas: on the one hand, the problem-specific mutations proved useful for the $(7+50)$-ES, indicating that we might not have make good usage of the domain knowledge; on the other hand, the $(7+50)$-ES (with problem-specific mutation) outperformed the $(1+7)$-ES when the initial population was not limited to modified Gibbs meshes: our hope is that starting from totally different parts of the search space could provide much better results in some particular situations ... which still remain to be 
identified. But in those yet hypothetical cases, the relevance of the evolutionary approach for the MNP would be clear.

\section{References}

1. T. Bäck and M. Schütz. Evolution strategies for mixed-integer optimization of optical multilayer systems. In J. R. McDonnell, R. G. Reynolds, and D. B. Fogel, editors, Proc. of the $4^{\text {th }}$ Annual Conf. on Evolutionary Programming. MIT Press, March 1995.

2. T. Bäck and M. Schütz. Intelligent mutation rate control in canonical GAs. In Z. W. Ras and M. Michalewicz, editors, Foundation of Intelligent Systems 9th Intl Symposium, ISMIS '96, pages 158-167. Springer Verlag, 1996.

3. P. G. Ciarlet. Mathematical Elasticity, Vol I : Three-Dimensional Elasticity. NorthHolland, Amsterdam, 1978.

4. B. Freisleben and P. Merz. New genetic local search operators for the TSP. In H.-M. Voigt, W. Ebeling, I. Rechenberg, and H.-P. Schwefel, editors, Proc. of the $4^{\text {th }}$ Conf. on Parallel Problems Solving from Nature, LNCS 1141, pages 890-899. Springer Verlag, 1996.

5. P.L. George. Automatic mesh generation, application to Finite Element Methods. Wiley \& Sons, 1991.

6. M. George-Schleuter. Asparagos96 and the travelling salesman problem. In T. Bäck, Z. Michalewicz, and X. Yao, editors, Proc. of the $4^{\text {th }}$ IEEE Intl Conf. on Evolutionary Computation, pages 171-174. IEEE Press, 1997.

7. F. Glover. Heuristics for integer programming using surrogate constraints. Decision Sciences, 8(1):156-166, 1977.

8. F. Glover and G. Kochenberger. Critical event tabu search for multidimensional knapsack problems. In Proc. of the Intl Conf. on Metaheuristics for Optimization, pages 113-133. Kluwer Publishing, 1995.

9. G.H. Golub and C. F. van Loan. Matrix Computations. John Hopkins University Press, 1996 - Third edition.

10. J. J. Grefenstette. Incorporating problem specific knowledge in genetic algorithms. In Davis L., editor, Genetic Algorithms and Simulated Annealing, pages 42-60. Morgan Kaufmann, 1987.

11. C. Kane and M. Schoenauer. Genetic operators for two-dimensional shape optimization. In J.-M. Alliot, E. Lutton, E. Ronald, M. Schoenauer, and D. Snyers, editors, Artificial Evolution, LNCS 1063. Springer Verlag, Septembre 1995.

12. P. Merz and B. Freisleben. Genetic local search for the TSP: New results. In T. Bäck, Z. Michalewicz, and X. Yao, editors, Proc. of the $4^{\text {th }}$ IEEE Intl Conf. on Evolutionary Computation, pages 159-164. IEEE Press, 1997.

13. M. Peyral, A. Ducoulombier, C. Ravisé, M. Schoenauer, and M. Sebag. Mimetic evolution. In Artificial Evolution'97, Springer Verlag. To appear.

14. N. J. Radcliffe and P. D. Surry. Fitness variance of formae and performance prediction. In L. D. Whitley and M. D. Vose, editors, Foundations of Genetic Algorithms 3, pages 51-72. Morgan Kaufmann, 1995.

15. M. Sebag, M. Schoenauer, and C. Ravisé. Toward civilized evolution: Developping inhibitions. In Th. Bäeck, editor, Proc. of the $7^{\text {th }}$ Intl Conf. on Genetic Algorithms. Morgan Kaufmann, 1997.

16. F. Sourd. Renumérotation de maillages par algorithmes génétiques, June 1996. Stage d'option de l'Ecole Polytechnique. 
17. D. Whitley, T. Starkweather, and D. Fuquay. Scheduling problems and travelling salesman: The genetic edge recombination operator. In J. D. Schaffer, editor, Proc. of the $3^{\text {rd }}$ Intl Conf. on Genetic Algorithms. Morgan Kaufmann, 1989.

18. O. C. Zienkiewicz. The Finite Element Method in Engineering Science. McGrawHill, New-York, 1st edition, 1967, 3rd edition, 1977. 